\title{
La fertilización mineral, orgánica y biológica sobre la producción de frijol común en Santa Rosa de Copán
}

Kevin J. Mejía ${ }^{1}$

\section{RESUMEN}

Con el objetivo de evaluar el efecto de la fertilización mineral, orgánica y biológica sobre la producción del frijol común (P. vulgaris L.) se desarrolló un ensayo durante los meses de febrero-mayo de 2015. El estudio se realizó en la finca El Padre, ubicada en el municipio de La Montañita, en el departamento de Copán, a $12 \mathrm{~km}$ de la ciudad de Santa Rosa de Copán. Se usó el diseño de bloque completamente al azar (BCA), con ocho tratamientos y cuatro repeticiones por cada uno, para un total de 32 unidades experimentales (UE). Las UE son parcelas de $8 \mathrm{~m} 2$ (4 hileras a $0.45 \mathrm{~m}$ y 5 $m$ de longitud). Los fertilizantes empleados fueron 12-24-12, urea $46 \%$ y KCl $60 \%$, combinados en fórmula de acuerdo al análisis de suelos. Lombricompost a razón de 4 ton.ha-1 depositado a fondo de surco; hongos micorriza por el método de peletizado de semilla. Seguidamente, se hizo combinación entre estos. La variedad empleada fue el Amadeus-77 (color rojo brillante). En el peso de 100 granos existió diferencia significativa, las semillas del tratamiento con compost lograron mayor peso $(27.68 \mathrm{~g})$, con respecto al control $(22.61 \mathrm{~g})$. El efecto de los tratamientos no fue significativo para el crecimiento de la planta a los $65 \mathrm{DDS}$, área foliar y rendimientos, mientras que la combinación de compost + micorriza tuvo mayor número de legumbres por planta; el tratamiento de fertilizante + compost + micorriza fue el que tuvo mayor número de granos por legumbre.

Palabras claves: fertilización, compost, micorriza, tratamientos.

\footnotetext{
${ }^{1}$ Beneficiario de una beca básica. Profesor del Departamento de Producción Vegetal, Centro Universitario Regional de Occidente CUROC,UNAH: masmejia@gmail.com; kevin.jarod@unah.edu.hn
} 


\section{ABSTRACT}

The objective of the following investigation was to evaluate the effect of mineral, organic and biological fertilization on the production of common bean (Phaseolus vulgaris L.). A trial was developed during the months of February to May 2015. The study was conducted on the farm "El Padre", in the municipality of "Montañita" ,province of Copán, located at $12 \mathrm{~km}$ from the city of Santa Rosa de Copán. A random block design (BCA) was used with eight treatments and four repetitions each for a total of 32 experimental units (EU). The EU consisted in 8 square meters lots each (4 rows to $0.45 \mathrm{~m}$ and $5 \mathrm{~m}$ in length). The fertilizer used were $12-24-12,46 \%$ of urea and $60 \%$ of $\mathrm{KCl}$ combined into a soil analysis formula. A combination of Vermicompost at 4 ton.ha-1 deposited at the bottom and mycorrhizal fungi pelleted seed was used (the bright red variety called Amadeus-77). There was significant difference in the weight of 100 grains, because the compost seeds treatment achieved greater weight (27.68 g) with respect to the control $(22.61 \mathrm{~g})$. The effect of the treatments was not significant for the growth of the plant at 65 DDS, leaf area and yield, while the combination of compost + micorriza had greater number of legumes per plant. The fertilizer + compost + micorriza treatment was the one with the largest number of grains per pod

Keywords: fertilization,, compost, micorriza, treatment. 


\section{INTRODUCCIÓN}

En Honduras, el cultivo del frijol (Phaseolus vulgaris L.) ocupa el segundo lugar en la producción de granos básicos y ocupa el $3 \%$ del PIB agropecuario (CEPAL, 2011). También representa el $20.25 \%$ del área dedicada a cultivos de consumo interno y anualmente se producen 88,700 toneladas, con rendimientos de 0.9 toneladas por hectárea (CEPAL, 2011).

El frijol es la fuente principal de proteínas para la mayoría de la población rural y urbana de escasos recursos de Centroamérica y el Caribe (Campoverde, 2004). En la región, el cultivo está en manos de pequeños agricultores, los cuales tienen limitado acceso a insumos, tierras fértiles y mecanización (SAG, 2014).

La fertilización es una de las prácticas que más ha progresado en las últimas décadas, ya que constituye uno de los pilares fundamentales de la producción agrícola. Actualmente no se concibe la explotación agrícola sin una adecuada fertilización que permita obtener del suelo toda su capacidad productiva (Domínguez, 1997). Brady y Weil (1999) mencionan que el manejo de los nutrientes en el suelo debe tener cuatro objetivos: costo efectivo para producir plantas de calidad, mantener la calidad del suelo, uso y conservación eficiente del recurso nutrientes y proteger el medioambiente.

Los agricultores de pequeña escala de Santa Rosa de Copán dedicados a la producción de granos básicos, sobre todo los productores en laderas, no logran rendimientos que les sirvan más allá que para su consumo, esto anidado a la baja tecnología que no alcanzan porcentajes superiores a 0.6 ton.ha-1 (USAID, 2013), en muchos casos por la forma de la fertilización que realizan al cultivo, es por ello que se vuelve importante probar dosis y tipos de fertilizantes que estén al alcance de una agricultura social aceptable, sana ambiental y económicamente viable.

Utilizando fertilizantes minerales aumentan el ciclo de nutrientes, pero afectan las propiedades físicas; mientras que la utilización de fertilizantes orgánicos mejora la calidad del suelo y proporcionan nutrientes a la planta, pero la liberación es mucho más lenta en comparación con los minerales, razón por la que muchos agricultores no los emplean, mientras que los fertilizantes biológicos ayudan a la planta a expandir su sistema radical y, por ende, hacer un mayor y mejor aprovechamiento de los nutrientes del suelo (Carvajal, 2010). 
Es por ello que se vuelve necesario el desarrollo de nuevas tecnologías y productos que estén más acordes con los pequeños productores, capaces de adaptarse a sus sistemas de producción, al tiempo que les resulten económicamente viables, socialmente aceptables, ecológicamente sanos y tecnológicamente adaptables (SAG, 2014).

Para el logro de la investigación se han establecido los siguientes objetivos:

\section{Objetivo general}

Evaluar el efecto de la fertilización mineral, orgánica y biológica sobre la producción del frijol común (P. vulgaris L.) en Santa Rosa de Copán durante los meses de febrero-mayo de 2015.

\section{Objetivos específicos}

1. Evaluar el efecto de la fertilización mineral, orgánica y biológica sobre las variables morfofisiológicas del cultivo de frijol común (P. vulgaris $L$ ).

2. Evaluar el efecto de la fertilización mineral, orgánica y biológica sobre los componentes del rendimiento del frijol común (P. vulgaris $L)$.

\section{MATERIALES Y MÉTODOS}

El estudio se realizó en la finca El Padre, ubicada en el municipio de La Montañita en el departamento de Copán, a 12 km de la ciudad de Santa Rosa de Copán. El lugar se encuentra a una altura de $910 \mathrm{msnm}$, con una temperatura promedio anual de $24 \pm 2{ }^{\circ} \mathrm{C}$ y una precipitación anual de $1100 \mathrm{~mm}$; el ensayo se realizó en el período comprendido entre los meses de febrero-mayo de 2015.

La variedad de frijol empleada fue Amadeus-77 (color rojo brillante) proporcionada por la Secretaría de Agricultura y Ganadería (SAG). Las evaluaciones se realizaron semanalmente, tomando 10 plantas al azar por parcela.

Evaluación del efecto de la fertilización mineral, orgánica y biológica sobre las variables morfofisiológicas del cultivo

Las variables a evaluar fueron: 
1. Crecimiento de la planta a 35 y 65 DDS medido en centímetros.

2. Porcentaje de materia seca (\%MS).

3. Área foliar (dm2).

Evaluación el efecto de la fertilización mineral, orgánica y biológica sobre los componentes del rendimiento

Variables medidas en el componente de rendimiento:

1. Número de legumbre por planta (número)

2. Número de semillas por legumbre (número)

3. Peso seco de 100 semillas $(g)$

4. Rendimiento (kg.ha-1)

\section{Tratamientos}

Se analizaron 32 unidades experimentales comprendidas por 8 tratamientos y 4 repeticiones por cada uno (ver cuadro 1).

Cuadro 1. Tratamientos empleados para evaluar el efecto de la fertilización sobre el desarrollo vegetativo y rendimiento en frijol común (P. vulgaris)

\begin{tabular}{llll}
\hline No. & Tratamientos & No. & Tratamientos \\
\hline 1 & Control & 5 & Fertilizante + micorriza \\
2 & Fertilizante & 6 & Fertilizante + compost \\
3 & Micorriza & 7 & Compost + micorriza \\
4 & Lombricompost & 8 & Fertilizante + micorriza + compost \\
\hline
\end{tabular}

Fuente: propia

En los tratamientos en los que se empleó el fertilizante mineral se usaron dosis básicas de producción basadas en el resultado del análisis de suelos. Con los tratamientos con micorriza se peletizó la semilla de frijol a una dosis de $1 \mathrm{~kg}$ por $45 \mathrm{~kg}$ de semilla; para la peletización se usó de adherente Pegol 101.

A los tratamientos basados en compost se les aplicó una dosis de 4 t.ha-1 colocándola en el fondo del surco. Las labores agrotécnicas aplicadas al cultivo únicamente estuvieron relacionadas con la eliminación de malezas y un riego semanal. En el manejo fitosanitario se aplicó Amistar 50 WG a una dosis de 0.7 L.ha-1 para evitar problemas por enfermedades; no se hizo control de plagas, debido a que estas no 
superaron el umbral económico (UE). La cosecha se realizó a partir de los 80 días después de sembrado, una vez alcanzada la madurez fisiológica del grano.

\section{Análisis de suelos}

Previamente al ensayo se realizó un análisis de suelo a una profundidad de $30 \mathrm{~cm}$. El pH del suelo fue fuertemente ácido (4.43), razón por la que se debió corregir con la aplicación de cal dolomítica, usada 30 días antes de la siembra, a una dosis recomendada de 7 t.ha-1.

\section{Preparación del lote}

La preparación del suelo se hizo con un pase de arado de disco y dos pases de grada liviana, dejando el suelo lo suficientemente mullido para la siembra; la siembra se realizó de forma manual con ayuda de estudiantes de la Carrera Técnico en Producción Agrícola del CUROC.

\section{Diseño experimental}

El experimento es de tipo unifactorial y para ello se usó un diseño de bloque completamente al azar (BCA). La unidad experimental (UE) eran parcelas de $8 \mathrm{~m} 2$ (4 hileras a $0.45 \mathrm{~m}$ y $6 \mathrm{~m}$ de longitud). Para reducir el error del experimento por el efecto de borde, en la toma de datos y la cosecha, se consideró como área útil las hileras centrales recortadas a $4 \mathrm{~m}$.

\section{Análisis estadístico}

El análisis de los datos se realizó con el software estadístico STATGRAPHICS Centurion versión 17.1.02 (32-bit), corrido en Windows 7. Se realizó un análisis de varianza (one-way ANOVA) y para contrastar la hipótesis nula de que ningún fertilizante tiene diferencia significativa sobre el rendimiento, se hizo una separación de medias con la prueba de Duncan a una $\mathrm{P} \leq 5 \%$ (5\% grados de libertad).

\section{RESULTADOS Y DISCUSIÓN}

Evaluación del efecto de la fertilización mineral, orgánica y biológica sobre las variables morfofisiológicas del cultivo de frijol común (P. vulgaris $L$ ) 
Las variables morfofisiológicas del cultivo permiten conocer cómo responde el cultivo ante la intervención de los tratamientos sobre este; para ello se considera la altura de la planta, el porcentaje de materia seca y el área foliar.

\section{Influencia de la fertilización sobre la altura de la planta a 35 y 65 DDS}

Las plantas evaluadas a los 35DDS (días después de siembra) mostraron diferencia significativa entre tratamientos (ver figura 1). El mayor crecimiento mostrado por las plantas fue para el tratamiento con fertilizante + micorriza $(48.1 \mathrm{~cm})$, mientras que el menor crecimiento lo mostró el control $(35.95 \mathrm{~cm})$; el resto de los tratamientos no mostraron diferencia, de los cuales se obtuvo un promedio general de $41.09 \mathrm{~cm}$. La evaluación de la altura de las plantas a los 65 DDS (en floración) no mostró diferencia significativa en su crecimiento, como promedio del tamaño de las plantas fue de $52.06 \mathrm{~cm}$ en los tratamientos.

Figura 1. Altura de la planta $(\mathrm{cm})$ a los 35 y 65 días después de sembrado (DDS)

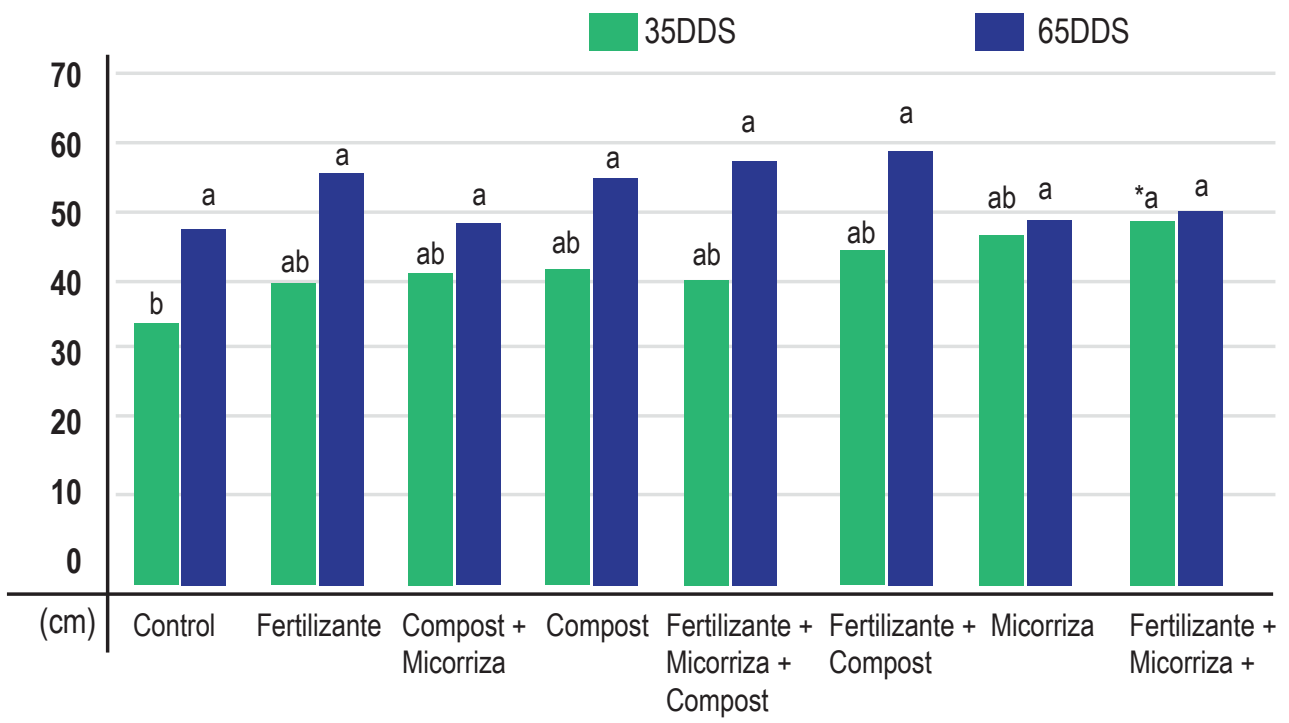

Fuente: propia

El mayor desarrollo presentado en las plantas de los tratamientos con micorriza se atribuye a que este tipo de fertilizante incrementa los procesos microbianos que benefician las plantas en breve tiempo, además de que producen sustancias estimuladoras del crecimiento vegetal (Valdéz, 2010). 


\section{Influencia de la fertilización en el porcentaje de materia seca}

La acumulación de materia seca es comúnmente usada como parámetro para caracterizar el crecimiento, porque usualmente tiene un gran significado económico. La producción de asimilados por las hojas y el punto hasta el cual pueden ser acumulados por el vertedero que representan los órganos que son cosechados, influencia significativamente el rendimiento del cultivo (Hammes, 2005).

En la muestra realizada para determinar el porcentaje de materia seca se encontró diferencia significativa, destacándose el tratamiento de fertilizante + micorriza del control (ver figura 2).

Figura 2. Influencia de la fertilización sobre el porcentaje de materia seca en el cultivo de frijol a 65 DDS

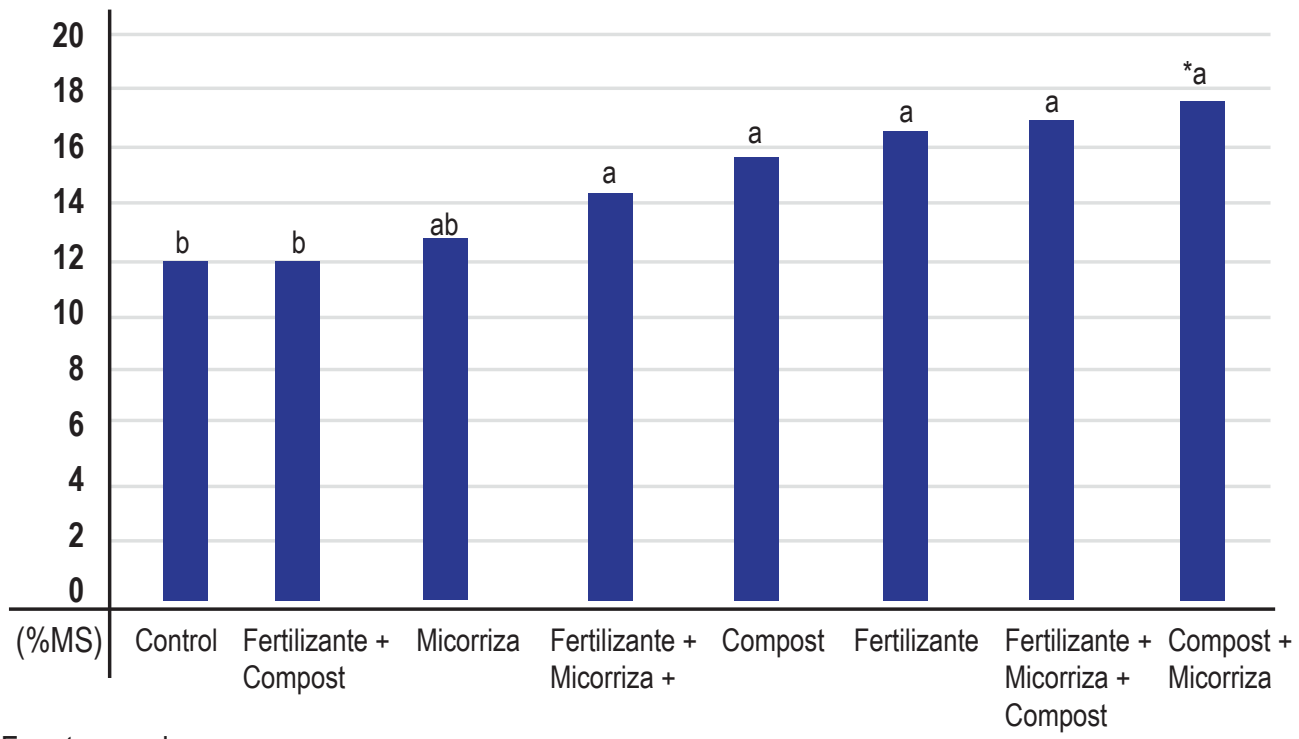

Fuente: propia

\section{Influencia de la fertilización sobre el área foliar}

La determinación del área foliar es fundamental en estudios de nutrición, crecimiento vegetal y aprovechamiento de nutrimentos, con esta variable se puede determinar la acumulación de materia seca, el metabolismo de carbohidratos, el rendimiento y calidad de cosecha. Es una medida necesaria para evaluar la intensidad de asimilación de las plantas, parámetro de gran relevancia cuando se efectúa el análisis de crecimiento del cultivo (Ruiz y otros, 2007). 
En la variable de área foliar de la planta (ver figura 3), los resultados no presentan diferencias significativas estadísticamente entre los tratamientos, sin embargo, como promedio general de los mismos se obtuvo $12 \mathrm{dm}^{2}$.

Figura 3. Área foliar de la planta de frijol a los 65 DDS

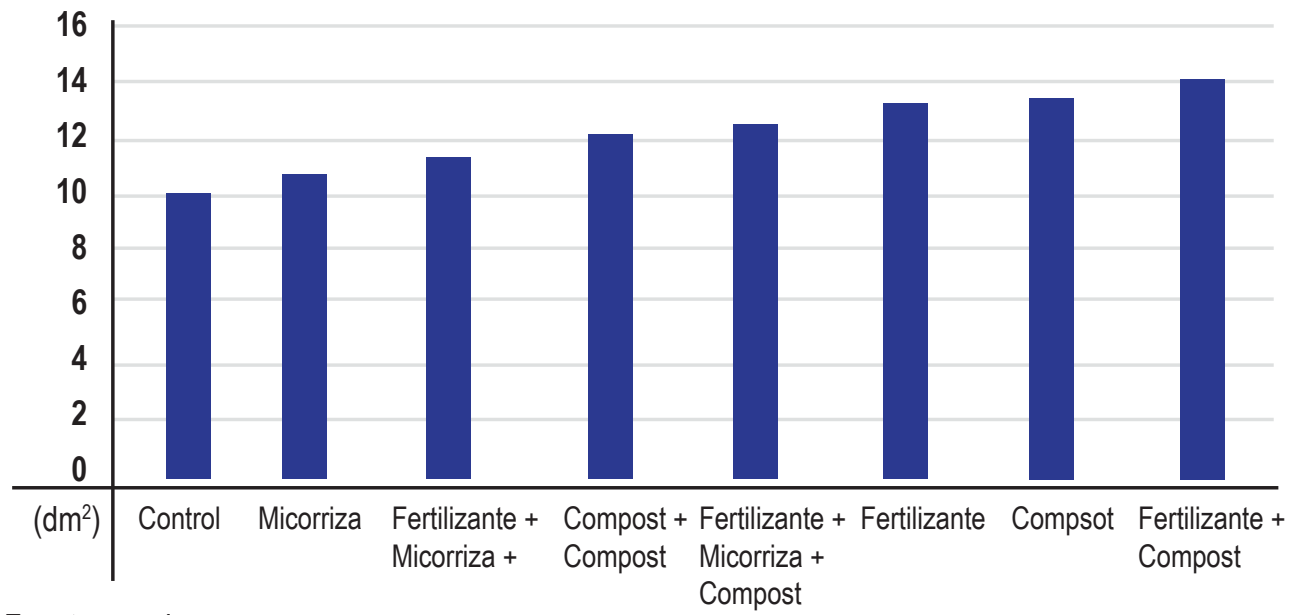

Fuente: propia

Los tratamientos fertilizante + compost y compost resultaron ser los que mayor área foliar alcanzaron, esto ocurrió debido a que este tipo de abonos orgánicos son de lenta liberación de nutrientes, generando como consecuencia que siempre existe disponibilidad para la planta, además del aporte que genera el fertilizante mineral, reflejado en este indicador.

Evaluación el efecto de la fertilización mineral, orgánica y biológica sobre los componentes del rendimiento del frijol común ( $P$. vulgaris $L$ )

Los componentes del rendimiento permiten tomar decisiones referentes a qué tratamientos o combinación de productos resultan más efectivos y prácticos de emplear en la producción, sin comprometer los rendimientos. Es por ello que se ha evaluado la influencia que han tenido los tipos de fertilización sobre el número de legumbres por planta, el número de granos por legumbre, el peso de 100 semillas expresado en gramos, rendimiento expresado en kilogramos por hectárea (kg.ha-1).

Influencia de la fertilización sobre el número de legumbres por planta

Con respecto al número de legumbres por planta (ver figura 4), existieron diferencias 
estadísticas significativas. Se observó que el tratamiento compost + micorriza, logró 18.5 legumbres por planta (4.75 legumbres más que el control). En el resto de los tratamientos fueron iguales estadísticamente, en los que se obtuvo como promedio 16.5 legumbres por planta.

Figura 4. Efecto de la fertilización sobre el número de legumbres por planta

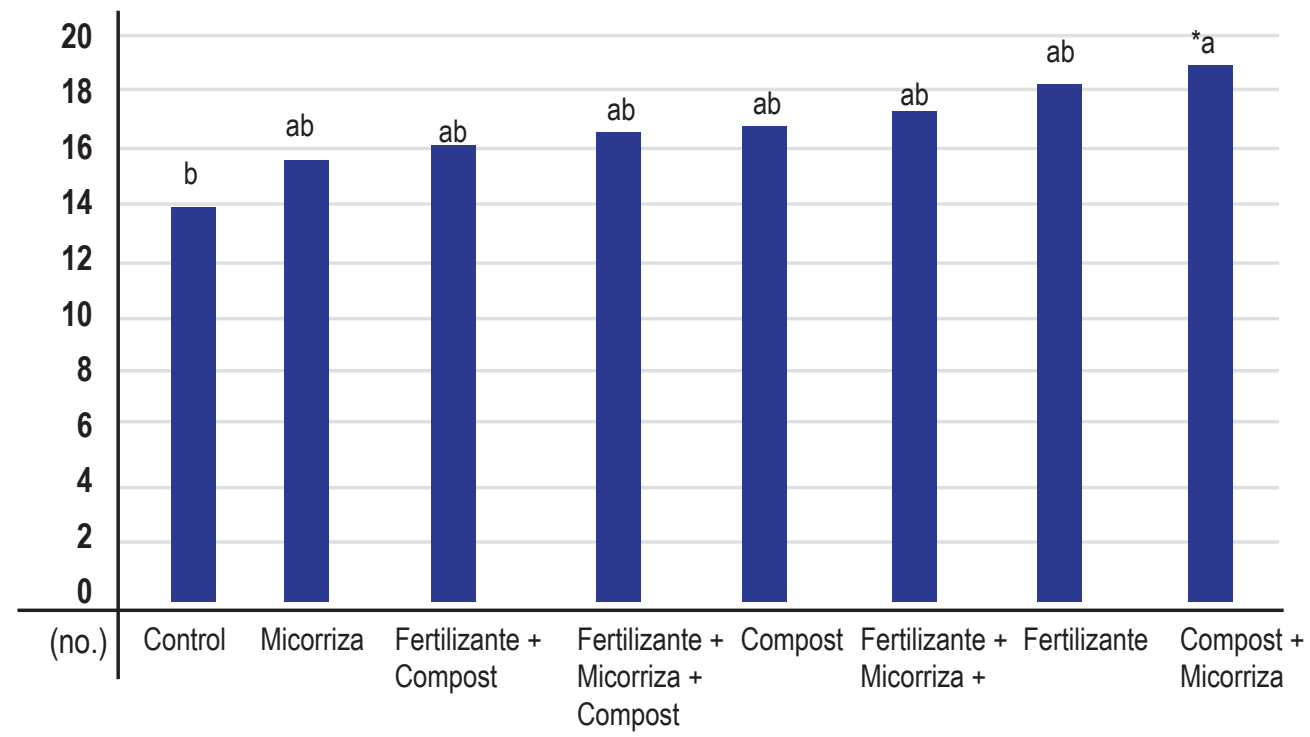

*Letras diferentes indican promedio diferente según Duncan $P \leq 0.05$.

Fuente: propia

Los resultados obtenidos en el tratamiento compost + micorriza, coinciden con lo realizado por Alvarado (2001), quien encontró que la micorriza influye en el incremento del número de legumbres por planta, no obstante, en combinación con compost para la investigación, resultó estadísticamente más efectivo.

\section{Influencia de la fertilización sobre el número de granos por legumbre}

Al analizar el número de granos por legumbre se refleja que existe diferencia significativa según $\mathrm{P} \leq 0.05$; siendo el tratamiento con fertilizante + compost + micorriza, combinados, el que presentó un promedio de 6.2 semillas por legumbre, con respecto al control 5.6 semillas por legumbre logradas en el control (ver figura 5). 
Figura 5. Efecto de la fertilización sobre el número de granos por legumbre

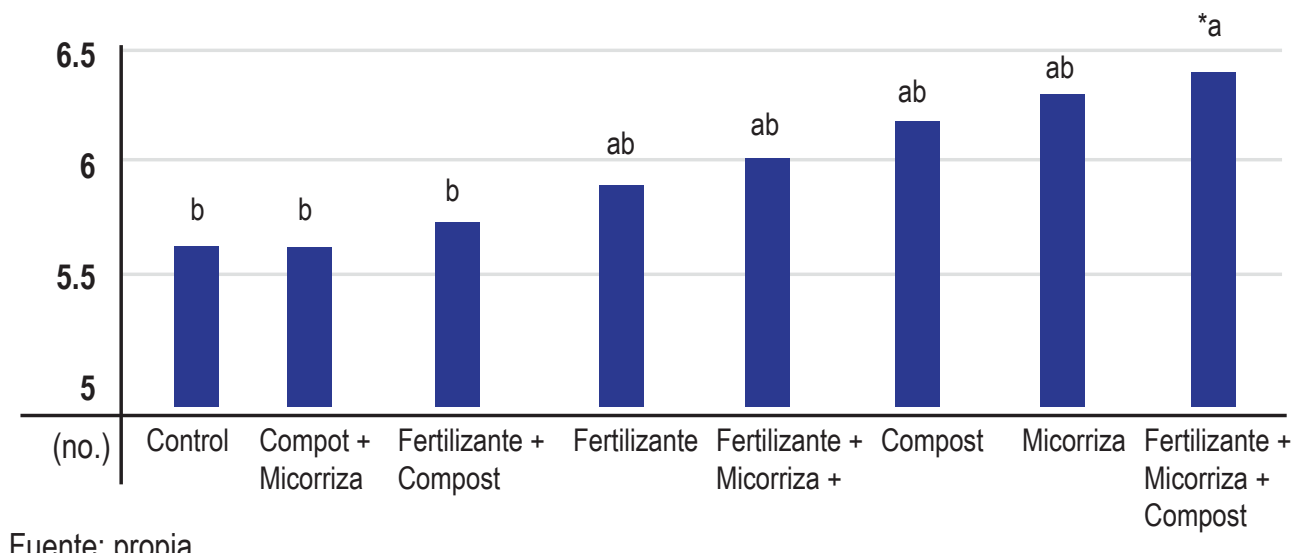

Fuente: propia

\section{Influencia de la fertilización sobre el peso de 100 granos}

En el peso de 100 granos existió diferencia significativa, aunque se observa que el tratamiento con compost es más pesado $(27.68 \mathrm{~g})$, con respecto al control $(22.61 \mathrm{~g})$, (ver figura 6).

Figura 6. Efecto de la fertilización sobre el peso de 100 granos

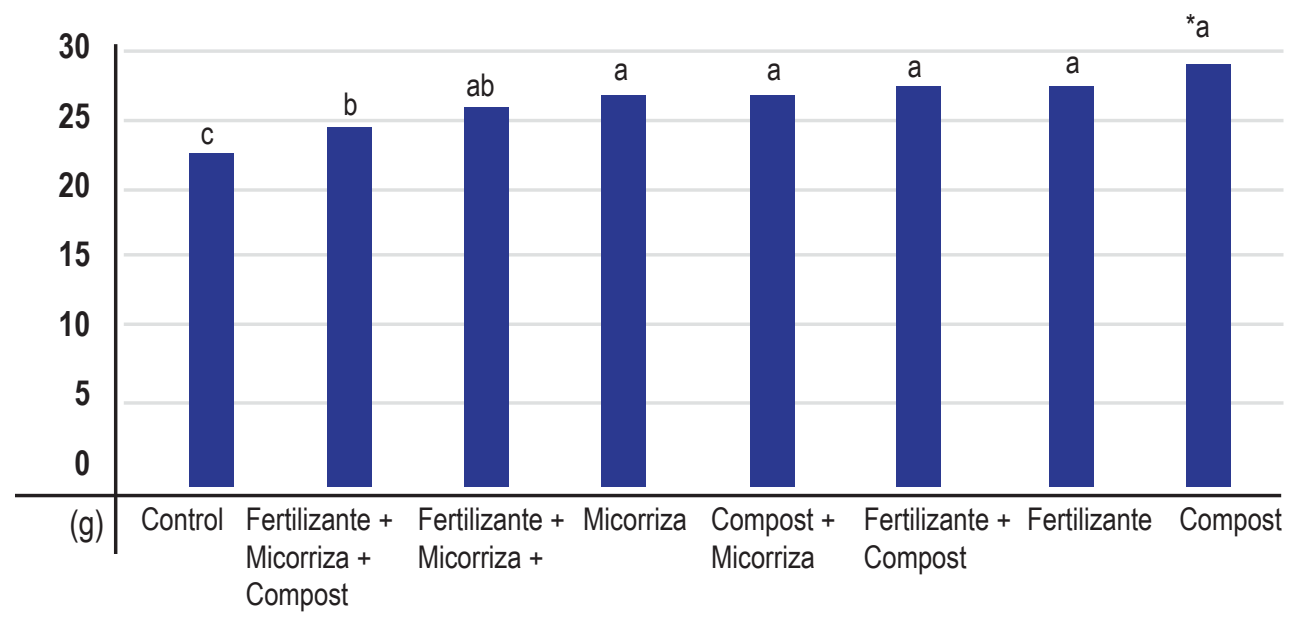

*Las letras iguales indican promedio iguales según Duncan $\mathrm{P} \leq 0.05$.

Fuente: propia 


\section{Influencia de la fertilización sobre el rendimiento del cultivo}

En la evaluación del rendimiento se tomaron todas las plantas de la parcela del tratamiento, los resultados obtenidos no presentaron diferencia significativa entre ellos (ver figura 7). Pese a ello, el tratamiento de fertilizante + compost, tuvo un rendimiento $300 \mathrm{~kg}$.ha-1 superior al control.

Figura 7. Efecto de la fertilización sobre el rendimiento de frijol expresado en kg.ha-1

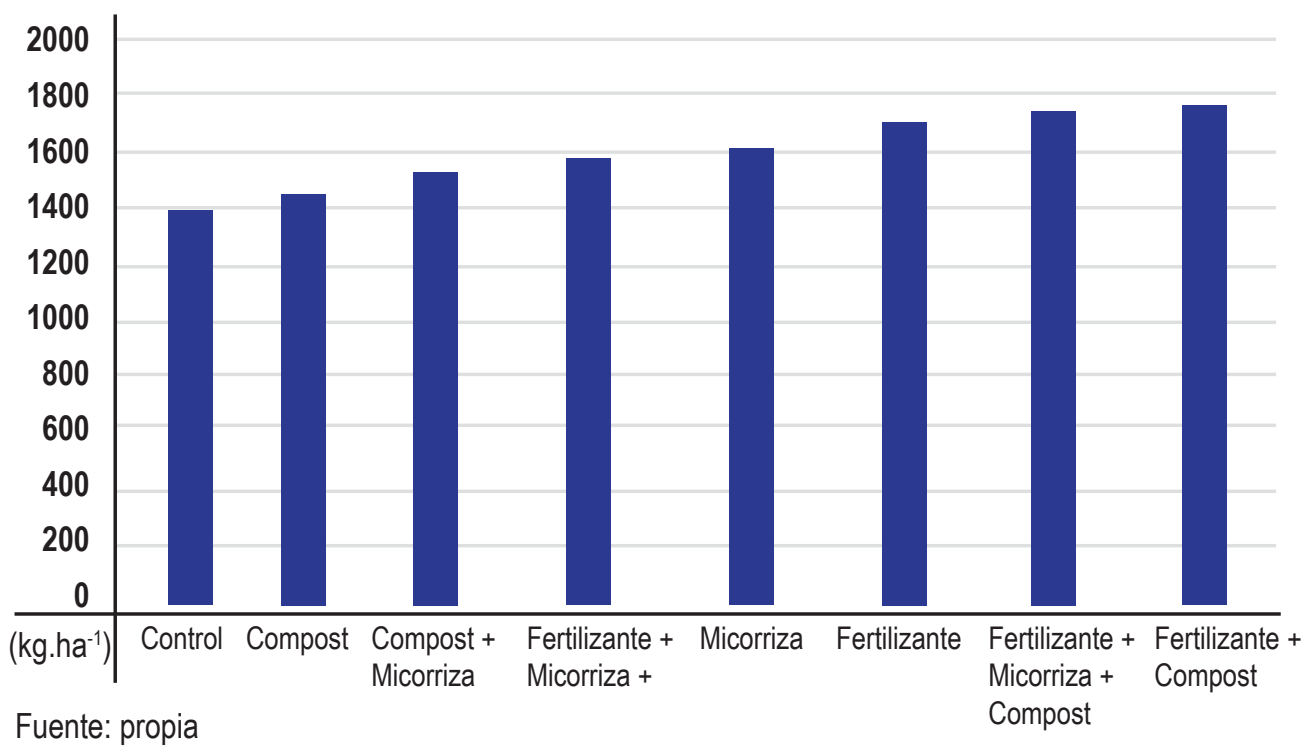

\section{Comparación económica}

A pesar de los rendimientos obtenidos en el ensayo, no mostraron diferencia significativa; el mayor gasto económico se realiza al utilizar la combinación de fertilizante + micorriza + compost, lo cual ha generado que los costos de producción superen los 35 mil lempiras por hectárea, que para pequeños productores no resulta rentable una inversión, no obstante, se recomienda que la aplicación de abonos orgánicos se haga paulatinamente en el transcurso de los años, con la finalidad de mantener la actividad microbiana en el suelo. 


\section{CONCLUSIONES}

1. El tratamiento de fertilizante + compost, mostró diferencia significativa con respecto al control en el desarrollo de la planta a los $35 \mathrm{dds}$, mientras que a los 65 dds no hubo diferencia.

2. La combinación de fertilizante + micorriza + compost, fue el tratamiento que tuvo mayor número de granos por legumbre; en el rendimiento ninguno de los tratamientos mostró diferencia.

\section{BIBLIOGRAFÍA}

Alvarado, G. G. (2001). Evaluación de los beneficios de la inoculación con micorriza arbuscular en el cultivo de frijol común (Phaseoulus vulgaris L). Tesis. Escuela Agrícola Panamericana Zamorano. Zamorano. Honduras.

Brady, N. and Weil, R. (1999). The nature and properties of soils. EE.UU.: Prentice Hall.

Campoverde, D. (2004). Respuesta de tres variedades de frijol a tres poblaciones y dos niveles de nitrógeno. Tesis. Escuela Agrícola Panamericana Zamorano. Zamorano. Honduras.

Carvajal, J. y Mera, A. (2010). Fertilización biológica: técnicas de vanguardia para el desarrollo agrícola sostenible. Producción + Limpia. Santa Marta. Colombia. . 5(2). $20 \mathrm{pp}$.

CEPAL. (2011). Subregión norte de América Latina y el Caribe: información del sector agropecuario, 2000-2010. México.

Díaz, M.; Saucedo, O.; Monzón, Y. y Rodríguez, G. (2005). Comparación del rendimiento de tres variedades de soya en la Estación Experimental Álvaro Barba Machado. Centro Agrícola, 36(4): 91-92 pp.

Domínguez, A. (1997). Tratado de fertilización. España: Mundi-Prensa.

Hammes, T. T. (2005). Growth and productivity of potato as influenced by cultivar and reproductivo growth II. Growth analysis, tuber yield and quality, 105(1), 29-44.

Ruiz, F.; Murillo, B.; García, J.; Troyo, E.; Palacios, A.; Beltran, A. y otros. (2007). Mediciones lineales en hoja para la estimación no destructiva del área foliar en albahaca (Ocimun basilicum L.). Universidad Autónoma de Chapingo, México: 13(1). 29-34 pp. 
USAID. (2013). Manual de producción del frijol. Proyecto USAID-ACCESO. La Lima, Cortés $25 \mathrm{pp}$.

Valdez, E. A. (2010). Empleo de abonos orgánicos y biofertilizantes en la reducción de afecciones por hongos patógenos del suelo y su repercusión en el incremento del rendimiento en el frijol común. Villa Clara: UCLV. 53 pp. 